\title{
( EDITOR'S CHOICE \\ Long-term neuroprotective effects of allopurinol after moderate perinatal asphyxia: follow-up of two randomised controlled trials
}

\author{
Joepe J Kaandorp, ${ }^{1}$ Frank van Bel, ${ }^{1}$ Sylvia Veen, ${ }^{2}$ Jan B Derks, ${ }^{1}$ Floris Groenendaal, \\ Monique Rijken, ${ }^{2}$ Elise Roze, ${ }^{3}$ Monica MA Uniken Venema, ${ }^{4}$ Carin MA Rademaker, ${ }^{5}$ \\ Arend F Bos, ${ }^{3}$ Manon JNL Benders ${ }^{1}$
}

\begin{abstract}
${ }^{1}$ Perinatal Center, Wilhelmina Children's Hospital, University Medical Center Utrecht, Utrecht, The Netherlands 2Department of Neonatology, Leiden University Medical Center, Leiden, The Netherlands

${ }^{3}$ Department of Neonatology, Beatrix Children's Hospital, University Medical Center Groningen, Groningen, The Netherlands

${ }^{4}$ Department of Medica Psychology, Wilhelmina Children's Hospital, University Medical Center Utrecht, Utrecht, The Netherlands ${ }^{5}$ Department of Clinical Pharmacy, Wilhelmina Children's Hospital, University Medical Center Utrecht, Utrecht, The Netherlands
\end{abstract}

\section{Correspondence to} Manon JNL Benders, Perinatal Center, University Medical Center Utrecht, Perinatal Center, Lundlaan 6 , KE 04.123.1, P0 box 85090, 3508 AB, Utrecht,

The Netherlands; m.benders@umcutrecht.nl

Received 16 May 2011 Accepted 12 October 2011 Published Online First 17 November 2011

\section{ABSTRACT}

Objective Free-radical-induced reperfusion injury has been recognised as an important cause of brain tissue damage after birth asphyxia. Allopurinol reduces the formation of free radicals, thereby potentially limiting the amount of hypoxia-reperfusion damage. In this study the long-term outcome of neonatal allopurinol treatment after birth asphyxia was examined.

Design Follow-up of 4 to 8 years of two earlier performed randomised controlled trials.

Setting Leiden University Medical Center, University Medical Center Groningen and University Medical Center Utrecht, The Netherlands.

Patients Fifty-four term infants were included when suffering from moderate-to-severe birth asphyxia in two previously performed trials.

Intervention Infants either received $40 \mathrm{mg} / \mathrm{kg}$ allopurinol (with an interval of $12 \mathrm{~h}$ ) starting within $4 \mathrm{~h}$ after birth or served as controls.

Main outcome measures Children, who survived, were assessed with the Wechsler Preschool and Primary Scales of Intelligence test or Wechsler Intelligence Scale for Children and underwent a neurological examination. The effect of allopurinol on severe adverse outcome (defined as mortality or severe disability at the age of 4-8 years) was examined in the total group of asphyxiated infants and in a predefined subgroup of moderately asphyxiated infants (based on the amplitude integrated electroencephalogram).

Results The mean age during follow-up $(n=23)$ was 5 years and 5 months (SD 1 year and 2 months). There were no differences in long-term outcome between the allopurinol-treated infants and controls. However, subgroup analysis of the moderately asphyxiated group showed significantly less severe adverse outcome in the allopurinol-treated infants compared with controls (25\% vs $65 \%$; RR $0.40,95 \% \mathrm{Cl} 0.17$ to 0.94 ).

Conclusions The reported data may suggest a (neuro) protective effect of neonatal allopurinol treatment in moderately asphyxiated infants.

Hypoxia-ischaemia (HI) during birth asphyxia damages the susceptible immature brain. Reperfusion of previously ischaemic brain tissue is now recognised as an important mechanism for substantial additional brain injury due to the formation of toxic free-radicals. ${ }^{12}$ This reperfusion injury may be ameliorated by neuroprotective strategies such as hypothermia with or without early postasphyxial pharmacological interventions such

\section{What is already known on this topic}

- Allopurinol reduces free-radical production, and in high dosages acts as a free-radical scavenger and free iron chelator.

- If birth asphyxia is too severe, neonatal allopurinol does not influence survival or short-term neurodevelopmental outcome, when administered postnatally.

\section{What this study adds}

- Neonatal allopurinol treatment may lower the risk of death or severe disability on the long term (at 4-8 years of age) in moderately asphyxiated infants.

- Neonatal treatment with high-dose allopurinol does not have negative side effects.

as allopurinol. ${ }^{3}$ Experimental studies investigating specific pharmacological therapies showed promising results, ${ }^{45}$ but appeared to be less successful in studies in the human neonates. ${ }^{6}$ Until now only therapeutic hypothermia of the brain or the whole body proved to reduce post-HI damage to the brain in moderately asphyxiated human neonates. ${ }^{7-10}$ In severely asphyxiated neonates (defined by a severely abnormal amplitude-integrated electroencephalogram (aEEG) plus seizures), hypothermia, however, did not seem to significantly improve the neurodevelopmental outcome. ${ }^{7}$

We previously performed two randomised controlled trials on the effect of high-dose allopurinol (40 $\mathrm{mg} / \mathrm{kg} /$ day) for reducing the post-HI brain damage in, respectively, 22 and 32 asphyxiated human neonates. ${ }^{11} 12$ The xanthine oxidase inhibitor allopurinol potentially protects against reperfusion-induced brain injury by reducing freeradical formation, and in high dosages also acts as a free-radical scavenger and free iron chelator. ${ }^{41314}$ A recent Cochrane meta-analysis, involving our two studies and a comparable Turkish trial, ${ }^{15}$ concluded that the currently available data are insufficient to determine whether allopurinol is beneficial as a neuroprotective treatment for birth asphyxia-induced HI encephalopathy. ${ }^{6}$ However, the 
neuroprotective effect of allopurinol in the subgroup of moderately asphyxiated children was not yet analysed.

In this study we investigated the neurodevelopmental and cognitive outcome between 4 and 8 years of age in the surviving patients of our two previously performed randomised controlled trials. Because recent studies showed that in particular infants suffering from moderate birth asphyxia benefit from neuroprotection with hypothermia in contrast to those suffering from severe birth asphyxia, we analysed differences in mortality and long-term developmental outcome between allopurinol-treated and non-treated children not only in the entire group of asphyxiated infants, but also in a subgroup of moderately asphyxiated infants. ${ }^{7}$

\section{METHODS}

\section{Participants}

The infants included in this follow-up study were the participants in our two randomised controlled trials concerning the effect of early neonatal allopurinol treatment in reducing the post-HI brain damage. ${ }^{11} 12$ The inclusion criteria of these studies were similar according to the following criteria: (near)term infants, without known chromosomal anomalies, who suffered from birth asphyxia defined as fetal distress (late decelerations on fetal monitoring or meconium staining; the need for resuscitation for $>2 \mathrm{~min}$; cord or lowest $\mathrm{pH}<7.00$ and multi-organ failure).

After admission, the brain electrical activity was monitored using aEEG (CFM, Lectromed; Oxford Instruments, Oxford, UK). The aEEG has been proven to be of value for evaluating background and seizure activity. This type of background pattern predicts long-term outcome. ${ }^{1617}$ The following patterns of increasing abnormalities can be distinguished into continuous normal voltage, discontinuous normal voltage, burst suppression, continuous extremely low voltage and flat trace. The last three patterns, which are abnormal, are induced by HI encephalopathy. Van Bel et al included all children with the abovementioned inclusion criteria, irrespective of the aEEG. In the study by Benders et al only those children, with the abovementioned inclusion criteria, which had a burst suppression pattern or worse on the aEEG were included.

\section{Intervention}

If all inclusion criteria were met, the infants received either two dosages of $20 \mathrm{mg} / \mathrm{kg}$ allopurinol (Apurin; Multipharma, Copenhagen, Denmark) intravenously starting within $4 \mathrm{~h}$ after birth (with an interval of $12 \mathrm{~h}$ ) or served as controls. Because high dosages were used for the first time, the study by van Bel et al was performed randomised, but not blinded to monitor possible side effects. Benders et al performed a randomised, double-blind and placebo-controlled study.

\section{Outcome assessment}

Children who survived and could be tested were assessed with the Wechsler Preschool and Primary Scale of Intelligence (WPPSI-III, an individually administered instrument for assessing the intelligence of children aged 2 years and 6 months to 7 years and 3 months) ${ }^{18}$ or the Wechsler Intelligence Scale for Children (WISC-III-NL for children aged 6-17 years) by psychologists. ${ }^{19}$ With these tests verbal, performance and full-scale IOs were measured. Total IOs were classified according to the guidelines of the Dutch Professional Association of Psychologists; IQ was defined normal if $\geq 90$, moderately delayed if the IQ ranged from $\geq 70$ to $<90$ and severely delayed if $<70 .{ }^{20}$
Every child underwent a standardised neurological examination by a neonatologist trained in neurodevelopmental assessments. Cerebral palsy (CP) was classified using the Gross Motor Function Classification System (GMFCS). The GMFCS describes the major functional characteristics of children with CP. It is a five-level pattern-recognition system. Children who are classified as GMFCS levels I and II have the potential to walk independently both indoors and outdoors, and in the community as well. In contrast, children classified into GMFCS levels III to $\mathrm{V}$ are limited in their self-mobility. They walk with a mobility device and are potential wheelchair users. ${ }^{21} 22$ The psychologists and neonatologists who assessed neurodevelopmental outcome were independent and not informed about patient allocation.

Neurodevelopmental outcome was classified into normal outcome, mild disability or severe disability. A full-scale IO of $\geq 90$ without any physical abnormalities was defined as normal. The CP classified as GMFCS level I or II, epilepsy with good response to treatment, hearing impairment with or without IQ ranges from $\geq 70$ to $<90$ were classified as mild disabilities. The CP classified as GMFCS levels III to V, epilepsy not responding to treatment, blindness, deafness with or without a full-scale IO $<70$ were regarded as severe disabilities.

The combined frequency of mortality or severe disability in survivors at 4-8 years of age was considered as severe adverse outcome.

Survival rates and long-term neurodevelopmental outcome were compared in both groups. All infants were then divided into two predefined groups for subgroup analysis based on the aEEG-signal; moderately asphyxiated neonates were defined by having a burst suppression pattern or better on the aEEG, and severely asphyxiated neonates were defined by having continuous low-voltage or flat-trace patterns on the aEEG. The analysis of the tracings was done by two neonatologists (F.v.B. and M.B.) with expertise in the interpretation of the aEEG. The aEEGs were classified using well-established criteria as published by Toet et al. ${ }^{16}$ This is a clinically used classification system to distinguish moderately asphyxiated infants from severely asphyxiated infants. ${ }^{17}$ Disagreements were solved by discussion. This subgroup analysis was performed to test the hypothesis that no advantage of neonatal allopurinol treatment is seen when brain damage is too severe.

\section{Statistical analyses}

Statistical analyses were performed with the SPSS 15.0 statistical package, version 15.0.1 (SPSS Inc, Chicago, IL, USA). Categorical variables were compared using Fisher's exact test. Differences regarding continuous data were assessed using Student's $t$ test or Mann-Whitney U test depending on the normality. The analyses concerning severe adverse outcome were stratified by trial. We report separate RR per trial and a pooled RR with 95\% CI, generated using a Mantel Haenszel approach (Review Manager (RevMan) (Computer program). Version 5.1. Copenhagen: The Nordic Cochrane Centre, The Cochrane Collaboration, 2011). Statistical significance was based on twosided tests with a cut-off level of 0.05 .

\section{Ethical approval}

Both studies were approved by the local ethical committees of the three participating hospitals (the Leiden University Medical Center, the University Medical Center Groningen and the University Medical Center Utrecht (The Netherlands). Informed consent was given by the parents of all participating children. 


\section{RESULTS}

Fifty-four full-term infants were included in the two previously performed studies: 22 children in the study by van Bel et al and 32 infants in the study by Benders et al. Twentyeight asphyxiated infants received allopurinol (ALLO) and 26 asphyxiated infants served as controls (CONT) (table 1). There were no significant differences between the groups regarding gestational age, birthweight, cord $\mathrm{pH}$, Apgar scores, distribution of aEEG patterns (table 2) or the age at follow-up.

In total, 31 infants died in the neonatal period, leaving 23 survivors (13 in the ALLO group and 10 in the CONT group) available for long-term follow-up. During the years it became clear that one child in the CONT group, with consanguine parents, was suspected to have a syndrome. The infant had mental retardation and epilepsy (not responding to antiepileptic treatment) which could not be solely explained by perinatal asphyxia since the neonatal MRI was normal. He was therefore excluded from further analyses.

The age of the children at follow-up was 5 years and 5 months (SD 1 year and 2 months) in the ALLO-treated and 5 years and 6 months (SD 1 year and 1 month) in the non-treated children. There were no infants with full-scale IQ scores lower than 70 as tested with the WISC or WPPSI. Full-scale IOs of $\geq 70$ and $<90$ were found in seven infants; three in the CONT group and four in the ALLO group. Hearing impairment was apparent in one child with normal IQ scores in the ALLO group. In the CONT group one child, with normal IQ scores, suffered from epilepsy well responded to treatment. In the ALLO group one child had developed a spastic quadriplegic CP classified as GMFCS level V with cortical blindness and severe mental retardation. One child in the CONT group developed a spastic quadriplegic CP classified as GMFCS level IV. Both children were not tested because severe neurodevelopmental delay was already established, and therefore the parents refused to cooperate.

One child in the ALLO group did not speak Dutch as his first language, so the verbal IQ could not be tested reliably. In this specific case the performal IQ, instead of the total IQ, was therefore used to classify outcome.

One child, in the ALLO group in the study by van Bel et al did not undergo the psychological test. The parents refused to cooperate, because the child did not have neurological problems at 5 years of age and received normal education. At the age of 12 all children in the Netherlands undergo a CITO test, ${ }^{23}$ which is a standardised Dutch test to assess the performance of children in different subareas (eg, mathematics and language). The outcome is a transformed scale score. The raw score (ie, the number of questions answered correctly) is converted to a percentile. Individual scores are compared with scores of children of the same age in the Netherlands. This child scored p70, which means $70 \%$ of all other children of that age who performed the same test scored lower. Her outcome was therefore classified as 'normal'.

No significant differences in long-term outcome were seen in children in the ALLO group compared with the CONT group (table 3). However, after excluding the most severely asphyxiated children based on the aEEG, significantly less severe adverse outcome was seen in the allopurinol-treated infants compared with controls ( $25 \%$ vs $65 \%$; $=0.047)$. Separate RR for severe adverse outcome per trial are shown in figure 1 . The pooled RR for severe adverse outcome in the ALLO group was $0.40(0.17-0.94)$ (table 3 , figure 1$)$.
Table 1 Survival rates of two randomised controlled trials concerning neonatal allopurinol after birth asphyxia

\begin{tabular}{llllll}
\hline & Van Bel et al & & & Benders et al & \\
\cline { 2 - 3 } & ALLO (n=11), & CONT (n=11), & & ALLO (n=17), $\mathbf{n}$ & CONT (n=15), $\mathbf{n}$ \\
\hline Died & 2 & 6 & 13 & 10 \\
Survivors & 9 & 5 & 4 & 5 \\
\hline
\end{tabular}

ALLO, allopurinol; CONT, controls.

Table 2 Baseline characteristics

\begin{tabular}{llll}
\hline & ALLO (n=28) & CONT (n=25)* & p \\
\hline Gestational age (weeks, days) & $40.0(37.0-41.4)$ & $39.6(36.0-42.0)$ & 0.253 \\
Birthweight (g) & $3500(2425-5270)$ & $3240(2300-4720)$ & 0.747 \\
Cord pH & $6.90(6.49-7.05)$ & $6.94(6.60-7.15)$ & 0.112 \\
Apgar score at 5 min & $4(2-7)$ & $4(1-8)$ & 0.471 \\
aEEG & & & \\
CNV, n (\%) & $8(29 \%)$ & $5(20 \%)$ & 0.405 \\
BS, n (\%) & $8(29 \%)$ & $12(48 \%)$ & 0.371 \\
CLV, n (\%) & $2(7 \%)$ & $4(16 \%)$ & 0.414 \\
FT, n (\%) & $10(36 \%)$ & $4(16 \%)$ & 0.109 \\
\hline
\end{tabular}

Data are reported as median (range), unless otherwise stated.

${ }^{*}$ One child was excluded because of a suspected syndrome.

ALLO, allopurinol; BS, burst suppression; CLV, continuous low voltage;

CNV, continuous normal voltage; CONT, controls; FT, flat trace.

\section{DISCUSSION}

Although no significant effect of neonatal allopurinol treatment is seen in reducing mortality or developmental disability in the overall group of asphyxiated infants, the present data suggest that neonatal treatment with allopurinol does lower the risk of severe adverse outcome (ie, mortality or severe disability) in moderately asphyxiated infants. These findings are in line with the hypothesis that no advantage of neonatal treatment is seen when birth asphyxia is too severe, as stated earlier by Gluckman et al when treating them with moderate hypothermia. ${ }^{7}$ However, the number of children involved in the analyses is small, thus the conclusions drawn have to be taken with some caution. There were also some small differences in inclusion criteria between both studies. The study by Benders et al only included children with a burst suppression pattern or worse on the aEEG. This might explain the differences in the overall mortality between the two studies, since it probably resulted in the inclusion of more severely asphyxiated infants in the study by Benders et al. Furthermore, there was a substantial variation between subjects in follow-up age, but since developmental scores calculated by WPPSI and WISC are adjusted for age, this should not be regarded as a big drawback.

In a true intention-to-treat analysis, the infant who was excluded because of a suspected syndrome should have been included. We nevertheless decided to exclude this child from further analyses. The child was diagnosed with epilepsy not responding to treatment, so would therefore be classified in the 'severe adverse outcome' group. With a small sample size, like in this study, including this child could have had enormous influence on the final results and conclusions. Because the child made part of the control group, this would have made the results more significantly in favour of allopurinol, which could not be justified.

It must be stated that there are limitations of aEEG interpretation depending on experience, especially in interpreting baseline drift, seizure recognition (with or without seizure detection), and the fact that it is only possible to give a general and not localised assessment of background pattern of brain activity. However, in our opinion, this method to assess the 
Table 3 Mortality and developmental outcome in survivors of two RCTs concerning neonatal allopurinol after birth asphyxia

\begin{tabular}{|c|c|c|c|c|c|c|c|}
\hline & \multicolumn{3}{|c|}{ Total group of asphyxiated infants } & \multicolumn{4}{|c|}{ Subgroup of moderately asphyxiated infants* } \\
\hline & $\operatorname{ALLO}(n=28)$ & CONT $(n=26)$ & $\mathbf{p}$ & ALLO $(n=16)$ & CONT $(n=17)$ & $\mathbf{p}$ & RR $(95 \%$ Cl) \\
\hline \multicolumn{8}{|l|}{ Mortality, n (\%) } \\
\hline Survivors & $13(46 \%)$ & $10(39 \%)$ & & $12(75 \%)$ & $7(41 \%)$ & & \\
\hline Excluded & 0 & $1 \dagger$ & & & & & \\
\hline Performance & $94.3(13.6)$ & $94.0(16.4)$ & 0.957 & $94.3(13.6)$ & $99.8(13.9)$ & 0.470 & \\
\hline Full Scale & $92.8(13.8)$ & $96.6(15.7)$ & 0.590 & $92.8(13.8)$ & $98.7(14.3)$ & 0.429 & \\
\hline Long-term neurodevelopmental outcome, $\mathrm{n}(\%)$ & $(n=13)$ & $(n=9)$ & & $(n=12)$ & $(n=7)$ & & \\
\hline Severe disabled§ & $1(8 \%)$ & $1(11 \%)$ & 1.000 & $0(0 \%)$ & $1(14 \%)$ & 0.368 & \\
\hline Mild disabled & $5(39 \%)$ & $4(44 \%)$ & 1.000 & $5(42 \%)$ & $2(29 \%)$ & 0.656 & \\
\hline
\end{tabular}

*Subgroup of moderately asphyxiated children; children with a burst suppression pattern or better on the aEEG.

tExcluded because of suspected syndrome.

$\ddagger$ Values are reported as mean (SD).

$\S$ The two children with a severe disability, both had CP (GMFCS $\geq 3$ ).

IThe group of mild disabled children included children with epilepsy well responding to treatment, hearing impairment or $10 \geq 70$ to $<90$.

**Normal outcome: $10 \geq 90$, no physical impairment.

††Severe adverse outcome was defined as mortality or severe disability.

$\ddagger \ddagger$ Pooled RR, generated using a Mantel Haenszel approach to stratify by trial.

ALLO, allopurinol; CONT, controls.

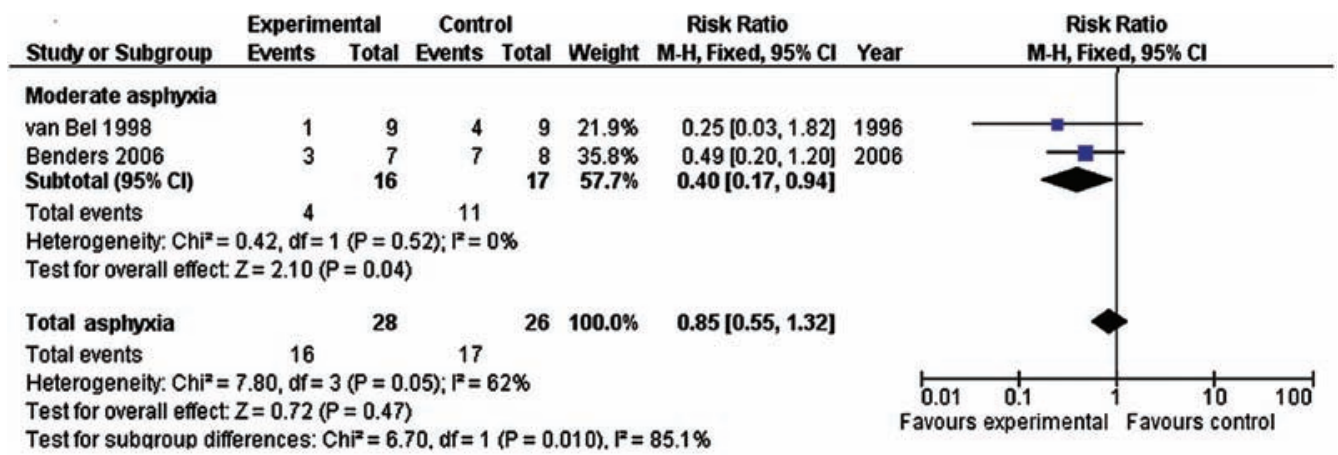

Figure 1 Severe adverse outcome in two randomised controlled trials concerning neonatal allopurinol after birth asphyxia. Occurrence of severe adverse outcome (indicated as 'events') in a subgroup of moderately asphyxiated infants, stratified by trial. A pooled RR for severe adverse outcome in the experimental (allopurinol) group was generated using a Mantel Haenszel approach.

severity of brain damage is reliable in both clinical care and research settings if used by well-trained neonatologists. ${ }^{17} 24$

To our knowledge, this is the first report concerning follow-up data after neonatal allopurinol treatment and, despite the above-mentioned limitations, we are of the opinion that this follow-up study provides us with some interesting data.

As already stated we could not find a neuroprotective effect of neonatal treatment with allopurinol in the overall group of asphyxiated infants such as is the case with hypothermia. This study, however, had insufficient power to exclude a modest but important effect size.

Another possible explanation might be the relatively late administration of allopurinol with a median of $3 \mathrm{~h}$ postnatally. Because of the vast amount of toxic free-radicals, an important reason for post-HI damage to the immature brain is produced during the $\mathrm{HI}$ event itself and in particular upon reperfusion and reoxygenation in the first 30 to 60 min after birth, we suggest that earlier treatment might be more effective. By intravenous administration of allopurinol to the mother when fetal distress, an important determinant of perinatal asphyxia, is suspected, therapeutic levels of allopurinol and its active metabolite oxypurinol can be reached even before birth. ${ }^{25} 26$ In this way, therapeutic levels of allopurinol are already available upon reperfusion, thereby reducing the formation of toxic free-radicals in an earlier and crucial stage, namely during maximal free-radical formation.

A recent pilot study in this respect, performed by our research group, showed a significant inversed correlation between the levels of fetal allopurinol and S100B (a clinically used marker for brain damage) in cord blood. ${ }^{26}$ A large multicenter randomised clinical trial on maternal treatment with 
allopurinol in case of signs of fetal hypoxia is now running in the Netherlands (NCT00189007) to investigate whether or not maternal allopurinol treatment can further improve the long-term outcome of neonates exposed to (severe) fetal hypoxia. ${ }^{27}$

In conclusion, the effects of neonatal treatment with allopurinol seem promising in moderately asphyxiated infants. Although the window of opportunities is probably quite small it is not clear whether the more severely asphyxiated children can also benefit from allopurinol treatment if treatment is started upon or even before birth in case of (severe) fetal hypoxia. Given the fact that superoxide production occurs upon and during the early reperfusion/reoxygenation phase, it seems to be appropriate to already treat the mother in case of suspected fetal distress during labour to reach therapeutic levels in the newborn before reoxygenation, thereby reducing the free-radical formation in the earliest possible state. Therapeutic hypothermia did not play any role in this follow-up study, because both initial trials were performed before the introduction of therapeutic mild hypothermia in clinical practice. For future research, however, it is very important to take the impact of mild therapeutic hypothermia on allopurinol metabolism and pharmacokinetics into account.

Contributors $\mathrm{FvB}, \mathrm{MB}, \mathrm{CR}$ and $\mathrm{FG}$ were involved in the conception and design of the study. MB, FvB, SV, MR, ER, AB and MUV had substantial contributions to the acquisition of the data. JK, FvB, MB, JD and CR analysed and interpreted the data. $\mathrm{JK}, \mathrm{FvB}$ and $\mathrm{MB}$ drafted the manuscript. All authors read, edited and approved the final manuscript for publication. They all had access to the study data that support the publication.

Ethics approval The local ethical committees of the Leiden University Medical Center, the University Medical Center Groningen and the University Medical Center Utrecht.

\section{Competing interests None.}

Provenance and peer review Not commissioned; externally peer reviewed.

\section{REFERENCES}

1. Ferriero DM. Neonatal brain injury. N Engl J Med 2004;351:1985-95.

2. McCord JM. Oxygen-derived free radicals in postischemic tissue injury. $N$ Eng/ J Med 1985:312:159-63.

3. van Bel F, Groenendaal F. Long-term pharmacologic neuroprotection after birth asphyxia: where do we stand? Neonatology 2008;94:203-10.

4. Palmer C, Towfighi J, Roberts RL, et al. Allopurinol administered afte inducing hypoxia-ischemia reduces brain injury in 7-day-old rats. Pediatr Res 1993;33:405-11.

5 Fan X, van Bel F. Pharmacological neuroprotection after perinatal asphyxia. J Matern Fetal Neonatal Med 2010;23 Suppl 3:17-19.

6 Chaudhari T, McGuire W. Allopurinol for preventing mortality and morbidity in newborn infants with suspected hypoxic-ischaemic encephalopathy. Cochrane Database Syst Rev 2008;2:CD006817.
7. Gluckman PD, Wyatt JS, Azzopardi D, et al. Selective head cooling with mild systemic hypothermia after neonatal encephalopathy: multicentre randomised trial. Lancet 2005;365:663-70.

8. Shankaran S, Laptook AR. Hypothermia as a treatment for birth asphyxia. Clin Obstet Gynecol 2007;50:624-35.

9. Edwards AD, Brocklehurst P, Gunn AJ, et al. Neurological outcomes at 18 months of age after moderate hypothermia for perinatal hypoxic ischaemic encephalopathy: synthesis and meta-analysis of trial data. BMJ 2010;340:c363.

10. Jacobs S, Hunt R, Tarnow-Mordi W, et al. Cooling for newborns with hypoxic ischaemic encephalopathy. Cochrane Database Syst Rev 2007:4:CD003311.

11. Van Bel F, Shadid M, Moison RM, et al. Effect of allopurinol on postasphyxial free radical formation, cerebral hemodynamics, and electrical brain activity. Pediatrics 1998;101:185-93.

12. Benders MJ, Bos AF, Rademaker CM, et al. Early postnatal allopurinol does not improve short term outcome after severe birth asphyxia. Arch Dis Child Fetal Neonatal Ed 2006;91:F163-5.

13. Peeters-Scholte C, van den Tweel E, Groenendaal F, et al. Redox state of near infrared spectroscopy-measured cytochrome aa(3) correlates with delayed cerebral energy failure following perinatal hypoxia-ischaemia in the newborn pig. Exp Brain Res 2004;156:20-6.

14. Russell GA, Cooke RW. Randomised controlled trial of allopurinol prophylaxis in very preterm infants. Arch Dis Child Fetal Neonatal Ed 1995;73:F27-31.

15. Gunes T, Ozturk MA, Koklu E, et al. Effect of allopurinol supplementation on nitric oxide levels in asphyxiated newborns. Pediatr Neurol 2007;36:17-24.

16. Toet MC, Hellström-Westas L, Groenendaal F, et al. Amplitude integrated EEG 3 and 6 hours after birth in full term neonates with hypoxic-ischaemic encephalopathy. Arch Dis Child Fetal Neonatal Ed 1999;81:F19-23.

17. Toet MC, Lemmers PM, van Schelven LJ, et al. Cerebral oxygenation and electrical activity after birth asphyxia: their relation to outcome. Pediatrics 2006;117:333-9

18. Wechsler D. Wechsler Preschool and Primary Scale of Intelligence. Third edition (WPPSI-III). San Antonio, TX: PsychCorp 2002

19. van Iterson L, Kaufman AS. Intra-individual subtest variability on the Dutch Wechsler Intelligence Scales for Children-Revised (WISC-R(NL)) for children with learning disabilities, psychiatric disorders, and epilepsy. Psychol Rep 2009;105:995-1008.

20. Resing W, Blok J. De classificatie van intelligentiescores: voorstel voor een eenduidig systeem. De Psycholoog 2002;37:244-8.

21. van Haastert IC, de Vries LS, Eijsermans MJ, et al. Gross motor functional abilities in preterm-born children with cerebral palsy due to periventricular leukomalacia. Dev Med Child Neurol 2008;50:684-9.

22. Palisano R, Rosenbaum $P$, Walter $S$, et al. Development and reliability of a system to classify gross motor function in children with cerebral palsy. Dev Med Child Neurol 1997;39:214-23.

23. Cito. Mission and Core values. http://www.cito.com/about cito/mission and core values.aspx (accessed September 2011)

24. de Vries LS, Hellström-Westas L. Role of cerebral function monitoring in the newborn. Arch Dis Child Fetal Neonatal Ed 2005;90:F201-7.

25. Boda D, Németh P, Kiss $P$, et al. Treatment of mothers with allopurinol to produce therapeutic blood levels in newborns. Prenat Neonat Med 1999:4:130-4.

26. Torrance HL, Benders MJ, Derks JB, et al. Maternal allopurinol during fetal hypoxia lowers cord blood levels of the brain injury marker S-100B. Pediatrics 2009;124:350-7.

27. Kaandorp JJ, Benders MJ, Rademaker CM, et al. Antenatal allopurinol for reduction of birth asphyxia induced brain damage (ALLO-Trial); a randomized double blind placebo controlled multicenter study. BMC Pregnancy Childbirth 2010;10:8 